\title{
A GENERALIZATION OF HERMITE INTERPOLATION
}

\author{
XIE-HUA SUN AND TINGFAN XIE \\ Department of Mathematics \\ China Institute of Metrology \\ Hangzhou, Zhejian 310034, PR CHINA
}

(Received December 4, 1992 and in revised form October 10, 1996)

ABSTRACT. We introduce a new interpolation at Chebyshev nodes. The usual Hermite interpolation is the limit case of our new interpolation as $h \rightarrow 0$

KEY WORDS AND PHRASES: Hermite interpolation, Chebyshev nodes, degree of approximation 1991 AMS SUBJECT CLASSIFICATION CODES: 41A05, 41A10, 41A25

\section{INTRODUCTION}

In many cases we do not know whether the interpolated function is differentiable or we only know the values of the interpolated function at nodes. Then, how do we construct an interpolation to describe the interpolated function better? In [1], the author introduced a new kind of interpolation $\left(0, \delta^{M}\right)$ interpolation $L_{n}^{\langle M\rangle}(f, x)$ for $2 \pi$-periodic function at nodes $x_{k}=2 k \pi / n(k=0,1, \ldots, n-1)$, such that

$$
\left\{\begin{array}{l}
L_{n}^{\langle M\rangle}\left(f, x_{k}\right)=f\left(x_{k}\right), \\
\delta^{M} L_{n}^{\langle M\rangle}\left(f, x_{k}\right)=\delta^{M} f\left(x_{k}\right)(k=0,1, \ldots, n-1),
\end{array}\right.
$$

where

$$
\begin{gathered}
\delta f(x)=\delta^{1} f(x)=f(x+h)-f(x-h), \\
\delta^{M} f(x)=\delta\left(\delta^{M-1} f(x)\right),(M \geq 2,0<h<\pi / n) .
\end{gathered}
$$

The usual Birkhoff and Hermite interpolations are the limit cases as $h \rightarrow 0$

From this it is natural to ask whether the similar interpolation problem of non-periodic function $b$, algebraic polynomials has a unique solution. If the answer is positive, what are the explicit formulae of fundamental polynomials? In this paper we will answer these questions for Chebyshev nodes in Sections 2 and 3 respectively In Section 4, we will consider the convergence.

\section{REGULARITY OF THE INTERPOLATION PROBLEM}

Let $T_{n}(x)=\cos n \theta(x=\cos \theta)$ be the Chebyshev polynomial and $x_{k}=\cos \theta_{k}=\cos (2 k-1) \pi /(2 n)$ $(k=1, \ldots, n)$ its zeros. Our interpolation problem is

P: For any two given sets of complex numbers $\left\{\alpha_{k}\right\}_{1}^{n}$ and $\left\{\beta_{k}\right\}_{1}^{n}$, whether or not there exists a unique algebraic polynomial $p_{2 n-1}(x)$ of order at most $2 n-1$ such that

$$
\left\{\begin{array}{l}
p_{2 n-1}\left(x_{k}\right)=\alpha_{k}, \\
\frac{p_{2 n-1}\left(\cos \left(\theta_{k}-h / 2\right)\right)-p_{2 n-1}\left(\cos \left(\theta_{k}+h / 2\right)\right)}{h \sin \theta_{k}}=\beta_{k},(k=1, \ldots, n) .
\end{array}\right.
$$

Taking a limit as $h \rightarrow 0$ in (2.1a), we have

$$
\left\{\begin{array}{l}
p_{2 n-1}\left(x_{k}\right)=\alpha_{k}, \\
p_{2 n-1}^{\prime}\left(x_{k}\right)=\beta_{k}, \quad(k=1, \ldots, n)
\end{array}\right.
$$

which are the conditions for Hermite interpolation Hence, this interpolation is a generalization of Hermite interpolation. 
We have the following

THEOREM 2.1. The interpolation problem $P$ always has a unique solution

PROOF. Set, for an algebraic polynomial $r_{2 n-1}$ of order at most $2 n-1$,

$$
r_{2 n-1}(\theta)=p_{2 n-1}(\cos \theta)=\frac{a_{0}}{2}+\sum_{j=1}^{2 n-1} a_{\jmath} \cos \jmath \theta
$$

Then $r_{2 n-1}$ is a cosine polynomial of order at most $2 n-1$.

It is obvious that Theorem 2.1 is equivalent to the system of equations

$$
\left\{\begin{array}{l}
r_{2 n-1}\left(\theta_{k}\right)=\frac{a_{0}}{2}+\sum_{\jmath=1}^{2 n-1} a_{\jmath} \cos j \theta_{k}=0, \\
\delta_{h / 2} r_{2 n-1}\left(\theta_{k}\right)=-2 \sum_{\jmath=1}^{2 n-1} a_{\jmath} \sin \frac{1}{2} j h \sin j \theta_{k}=0,(k=1, \ldots, n) .
\end{array}\right.
$$

From the first set of equations of (2.2)

$$
\begin{aligned}
r_{2 n-1}\left(\theta_{k}\right) & =\frac{a_{0}}{2}+\sum_{\jmath=1}^{n} a_{\jmath} \cos j \theta_{k}+\sum_{\jmath=1}^{n-1} a_{2 n-\jmath} \cos (2 n-j) \theta_{k} \\
& =\frac{a_{0}}{2}+\sum_{\jmath=1}^{n-1}\left(a_{\jmath}-a_{2 n-\jmath}\right) \cos j \theta_{k}=0, \quad k=1, \ldots, n
\end{aligned}
$$

(2 3) shows that the following trigonometric polynomial of order $\leq n-1$

$$
\frac{a_{0}}{2}+\sum_{j=1}^{n-1}\left(a_{\jmath}-a_{2 n-\jmath}\right) \cos j \theta
$$

has $2 n$ zeros in $[-\pi, \pi): \theta= \pm \theta_{k}(k=1, \ldots, n)$ Hence, we must have

$$
a_{0}=0, \quad a_{\jmath}-a_{2 n-\jmath}=0, \quad j=1, \ldots, n-1 .
$$

On the other hand, from the second set of equations in (2.2) it is easy to see that

$$
\begin{aligned}
\delta_{h / 2} r_{2 n-1}\left(\theta_{k}\right)= & -2\left\{\sum_{\jmath=1}^{n-1}\left(a_{\jmath} \sin \frac{1}{2} j h+a_{2 n-\jmath} \sin \frac{1}{2}(2 n-j) h\right) \sin \jmath \theta_{k}\right. \\
& \left.+a_{n} \sin \frac{1}{2} n h \sin n \theta_{k}\right\}=0 \quad k=1, \ldots, n .
\end{aligned}
$$

Therefore the trigonometric polynomial of order $\leq n$

$$
\sum_{j=1}^{n-1}\left(a_{\jmath} \sin \frac{1}{2} j h+a_{2 n-\jmath} \sin \frac{1}{2}(2 n-j) h\right) \sin j \theta+a_{n} \sin \frac{1}{2} n h \sin n \theta
$$

has $2 n+1$ zeros: $\theta=0 ; \theta= \pm \theta_{k}(k=1, \ldots, n)$. Hence, we have

$$
a_{n} \sin \frac{1}{2} n h=0, \quad a_{\jmath} \sin \frac{1}{2} j h+a_{2 n-\jmath} \sin \frac{1}{2}(2 n-j) h=0, \quad \jmath=1, \ldots, n-1 .
$$

Solving the system of (2.4) and (2.6) and observing that

$$
\sin \frac{1}{2} j h>0 \text { and } \sin \frac{1}{2}(2 n-j) h>0,
$$

we obtain

$$
a_{j}=0, \quad j=0,1, \ldots, 2 n-1 .
$$




\section{FUNDAMENTAL POLYNOMIALS OF THE INTERPOLATION}

Our main result is the following

THEOREM 3.1. The fundamental polynomials $p_{m}(x)$ and $q_{m}(x)$ satisfying the following conditions

$$
\left\{\begin{array}{l}
p_{m}\left(\cos \theta_{k}\right)=\delta_{k m} \\
\delta_{h / 2} p_{m}\left(\cos \theta_{k}\right)=0,(m, k=1, \ldots, n)
\end{array}\right.
$$

and

$$
\left\{\begin{array}{l}
q_{m}\left(\cos \theta_{k}\right)=0, \\
-\frac{\delta_{h / 2} q_{m}\left(\cos \theta_{k}\right)}{h \sin \theta_{k}}=\delta_{k m}, \quad(m, k=1, \ldots, n)
\end{array}\right.
$$

respectively, are given by

$$
p_{m}(x)=\frac{2}{n}\left(\frac{1}{2}+\sum_{j=1}^{2 n-1} \frac{\sin \frac{1}{2}(2 n-j) h \cos j \theta_{m}}{\sin \frac{1}{2} j h+\sin \frac{1}{2}(2 n-j) h} \cos j \theta\right),(x=\cos \theta)
$$

and

$$
q_{m}(x)=\frac{h \sin \theta_{m}}{n} \sum_{j=1}^{2 n-1} \frac{\sin j \theta_{m}}{\sin \frac{1}{2} j h+\sin \frac{1}{2}(2 n-j) h} \cos j \theta,(x=\cos \theta) .
$$

PROOF. First, we prove the polynomial $p_{m}(x)$ satisfies (3.1). Using the following identity

$$
\frac{2}{n}\left\{\frac{1}{2}+\sum_{j=1}^{n-1} \cos j \theta_{m} \cos j \theta_{k}\right\}=\delta_{k m}, \quad \theta_{k}=\frac{2 k-1}{2 n} \pi, \quad(k, m=1, \ldots, n) .
$$

we have

$$
\begin{aligned}
p_{m}\left(\cos \theta_{k}\right)= & \frac{2}{n}\left\{\frac{1}{2}+\sum_{j=1}^{n-1} \frac{\sin \frac{1}{2}(2 n-j) h \cos j \theta_{m}}{\sin \frac{1}{2} j h+\sin \frac{1}{2}(2 n-j) h} \cos j \theta_{k}\right. \\
& \left.+\sum_{j=1}^{n-1} \frac{\sin \frac{1}{2} j h \cos (2 n-j) \theta_{m}}{\sin \frac{1}{2} j h+\sin \frac{1}{2}(2 n-j) h} \cos (2 n-j) \theta_{k}\right\} \\
= & \frac{2}{n}\left\{\frac{1}{2}+\sum_{j=1}^{n-1} \frac{\sin \frac{1}{2}(2 n-j) h \cos j \theta_{m}}{\sin \frac{1}{2} j h+\sin \frac{1}{2}(2 n-j) h} \cos j \theta_{k}\right. \\
& \left.+\sum_{j=1}^{n-1} \frac{\sin \frac{1}{2} j h \cos j \theta_{m}}{\sin \frac{1}{2} j h+\sin \frac{1}{2}(2 n-j) h} \cos j \theta_{k}\right\} \\
= & \frac{2}{n}\left\{\frac{1}{2}+\sum_{j=1}^{n-1} \cos j \theta_{m} \cos j \theta_{k}\right\}=\delta_{k m} .
\end{aligned}
$$

And

$$
\begin{aligned}
\delta_{\frac{h}{2}} p_{m}\left(\cos \theta_{k}\right)= & -\frac{4}{n} \sum_{j=1}^{2 n-1} \frac{\sin \frac{1}{2}(2 n-j) h \cos j \theta_{m}}{\sin \frac{1}{2} j h+\sin \frac{1}{2}(2 n-j) h} \sin \frac{1}{2} j h \sin j \theta_{k} \\
= & -\frac{4}{n} \sum_{j=1}^{n-1} \frac{\sin \frac{1}{2} j h \sin \frac{1}{2}(2 n-j) h}{\sin \frac{1}{2} j h+\sin \frac{1}{2}(2 n-j) h} \cos j \theta_{m} \sin j \theta_{k} \\
& +\frac{4}{n} \sum_{j=1}^{n-1} \frac{\sin \frac{1}{2} j h \sin \frac{1}{2}(2 n-j) h}{\sin \frac{1}{2} j h+\sin \frac{1}{2}(2 n-j) h} \cos (2 n-j) \theta_{m} \sin (2 n-j) \theta_{k} \\
= & 0 .
\end{aligned}
$$

Similarly, using the identity 


$$
\frac{2}{n}\left\{\frac{1}{2} \sin n \theta_{m} \sin n \theta_{k}+\sum_{j=1}^{n-1} \sin j \theta_{m} \sin j \theta_{k}\right\}=\delta_{k m}, \quad k, m=1, \ldots, n,
$$

we can prove (3 4) satisfies (3 2) Q E D

\section{CONVERGENCE OF THE INTERPOLATION}

For $f \in C[-1,1]$ define the following operators

$$
S_{n}(f, x)=\sum_{k=1}^{n} f\left(x_{k}\right) p_{k}(x)-\sum_{k=1}^{n} \frac{f\left(\cos \left(\theta_{k}+\frac{h}{2}\right)\right)-f\left(\cos \left(\theta_{k}-\frac{h}{2}\right)\right)}{h \sin \theta_{k}} q_{k}(x)
$$

and

$$
s_{n}(f, x)=\sum_{k=1}^{n} f\left(x_{k}\right) p_{k}(x),
$$

which satisfy the following conditions

$$
\left\{\begin{array}{l}
S_{n}\left(f, x_{k}\right)=f\left(x_{k}\right) \\
\delta_{\frac{h}{2}} S_{n}\left(f, \cos \theta_{k}\right)=\delta_{\frac{h}{2}} f\left(\cos \theta_{k}\right) \quad(k=1, \ldots, n)
\end{array}\right.
$$

and

$$
\left\{\begin{array}{l}
s_{n}\left(f, x_{k}\right)=f\left(x_{k}\right) \\
\delta_{\frac{1}{2}} s_{n}\left(f, \cos \theta_{k}\right)=0 \quad k=1, \ldots, n
\end{array}\right.
$$

If we take limits in (4 1a)-(4 4a), we have for $f \in C^{1}[-1,1]$

$$
H_{n}(f, x)=\sum_{k=1}^{n} f\left(x_{k}\right) P_{k}(x)+\sum_{k=1}^{n} f^{\prime}\left(x_{k}\right) Q_{k}(x),
$$

and for $f \in C[-1,1]$

$$
F_{n}(f, x)=\sum_{k=1}^{n} f\left(x_{k}\right) P_{k}(x),
$$

which satisfies the following conditions

$$
\left\{\begin{array}{l}
H_{n}\left(f, x_{k}\right)=f\left(x_{k}\right) \\
H_{n}^{\prime}\left(f, x_{k}\right)=f^{\prime}\left(x_{k}\right), \quad(k=1, \ldots, n)
\end{array}\right.
$$

and

$$
\left\{\begin{array}{l}
F_{n}\left(f, x_{k}\right)=f\left(x_{k}\right) \\
F_{n}^{\prime}\left(f, x_{k}\right)=0, \quad(k=1, \ldots, n)
\end{array}\right.
$$

respectively. Obviously,

$$
\begin{aligned}
P_{k}(x) & =\lim _{h \rightarrow 0} p_{k}(x)=\frac{2}{n}\left\{\frac{1}{2}+\sum_{j=1}^{2 n-1}\left(1-\frac{j}{2 n}\right) \cos j \theta_{k} \cos j \theta\right\} \\
& =\frac{1}{4 n^{2}}\left[\left(\frac{\sin n\left(\theta-\theta_{k}\right)}{\sin \frac{1}{2}\left(\theta-\theta_{k}\right)}\right)^{2}+\left(\frac{\sin n\left(\theta+\theta_{k}\right)}{\sin \frac{1}{2}\left(\theta+\theta_{k}\right)}\right)^{2}\right]
\end{aligned}
$$

and 


$$
\begin{aligned}
Q_{k}(x) & =\lim _{h \rightarrow 0} q_{k}(x)=\frac{\sin \theta_{k}}{n^{2}} \sum_{j=1}^{2 n-1} \sin j \theta_{k} \cos j \theta \\
& =\frac{\sin ^{2} \theta_{k} \cos ^{2} n \theta}{n^{2}\left(\cos \theta-\cos \theta_{k}\right)} .
\end{aligned}
$$

Operators $H_{n}(f, x)$ and $F_{n}(f, x)$ are the famous Hermite and Hermite-Fejér operators respectively (see [2] and [3])

Now we state our main results in this section

THEOREM 4.1. If $c$ is a constant $(0<c<1)$ and $h=c \pi / n$, then we have for $f \in C[-1,1]$

$$
f(x)-S_{n}(f, x)=O\left(E_{2 n-1}(f) \log n\right),
$$

where $E_{n}(f)$ is the best approximation of $f$ by algebraic polynomials of order $\leq n$ and the sign " $O$ " is independent of $f, n$ and $x$

THEOREM 4.2. If $\rho$ is a constant $(0<\rho<1)$ and $0<h<\rho \pi / n$, then we have for $f \in C[-1,1]$

$$
f(x)-s_{n}(f, x)=O\left(\frac{1}{2}\right) \sum_{k=1}^{n} \omega\left(f, \frac{1}{k}+\frac{\sqrt{1-x^{2}}}{k^{2}}\right),
$$

where the sign " $O$ " is independent of $h, f, n$ and $x$

In order to prove the above theorems, we need some lemmas

LEMMA 4.1. The following estimates are valid.

$$
\sum_{k=1}^{n}\left|p_{k}(x)\right|=O(1)
$$

and

$$
\sum_{k=1}^{n} \frac{\left|q_{k}(x)\right|}{\sin \theta_{k}}=O\left(\frac{\log n}{n}\right) .
$$

We omit the proof since it is similar to the one in [1]

LEMMA 4.2. Let $1 \leq j \leq n$ satisfy that

$$
\inf _{1 \leq k \leq n}\left|\theta-\theta_{k}\right|=\left|\theta-\theta_{j}\right|
$$

and let $i=|k-j|$. Then

$$
\left|p_{k}(x)\right|=O\left(\frac{1}{i^{2}}\right),(k \neq j)
$$

and

$$
\left|p_{j}(x)\right|=O(1)
$$

PROOF. It is clear that

$$
\begin{aligned}
p_{k}(x)= & \frac{1}{2 n}\left(1+2 \sum_{j=1}^{2 n-1} \frac{\sin \frac{1}{2}(2 n-j) h}{\sin \frac{1}{2} j h+\sin \frac{1}{2}(2 n-j) h} \cos j\left(\theta-\theta_{k}\right)\right) \\
& +\frac{1}{2 n}\left(1+2 \sum_{j=1}^{2 n-1} \frac{\sin \frac{1}{2}(2 n-j) h}{\sin \frac{1}{2} j h+\sin \frac{1}{2}(2 n-j) h} \cos j\left(\theta+\theta_{k}\right)\right) \\
:= & I_{1}+I_{2} .
\end{aligned}
$$

Denote the Fejér kernel by 


$$
\sigma_{k}(x)=1+2 \sum_{j=1}^{k-1}\left(1-\frac{\jmath}{k}\right) \cos \jmath x=\frac{1}{k}\left(\frac{\sin \frac{1}{2} k x}{\sin \frac{1}{2} x}\right)^{2} .
$$

Then we have the following identity

$$
(j+1) \sigma_{\jmath+1}(x)-2 j \sigma_{\jmath}(x)+(j-1) \sigma_{\jmath-1}(x)=2 \cos \jmath x .
$$

By (4 12), we have

$$
\begin{aligned}
I_{1}= & \frac{1}{2 n}\left[1-2 \frac{\sin \frac{1}{2}(2 n-1) h}{\sin \frac{1}{2} h+\sin \frac{1}{2}(2 n-1) h} \sigma_{1}\left(\theta-\theta_{k}\right)\right. \\
& \left.+\frac{\sin (n-1) h}{\sin h+\sin (n-1) h} \sigma_{1}\left(\theta-\theta_{k}\right)\right] \\
& +\frac{1}{2 n}\left[\frac{\sin \frac{1}{2} h}{\sin \frac{1}{2} h+\sin \frac{1}{2}(2 n-1) h} 2 n \sigma_{2 n}\left(\theta-\theta_{k}\right)\right. \\
& +\frac{\sin h}{\sin h+\sin (n-1) h}(2 n-1) \sigma_{2 n-1}\left(\theta-\theta_{k}\right) \\
& \left.-\frac{2 \sin \frac{1}{2} h}{\sin \frac{1}{2} h+\sin \frac{1}{2}(2 n-1) h}(2 n-1) \sigma_{2 n-1}\left(\theta-\theta_{k}\right)\right] \\
& +\frac{1}{2 n} \sum_{j=2}^{2 n-2}\left(\frac{\sin \frac{1}{2}(2 n-j+1) h}{\sin \frac{1}{2}(j-1) h+\sin \frac{1}{2}(2 n-\jmath+1) h}\right. \\
& -\frac{2 \sin \frac{1}{2}(2 n-j) h}{\sin \frac{1}{2} j h+\sin \frac{1}{2}(2 n-j) h} \\
& \left.+\frac{\sin \frac{1}{2}(2 n-j-1) h}{\sin \frac{1}{2}(j+1) h+\sin \frac{1}{2}(2 n-j-1) h}\right) j \sigma_{\jmath}\left(\theta-\theta_{k}\right) \\
:= & I_{11}+I_{12}+I_{13} .
\end{aligned}
$$

It is obvious that

$$
I_{11}=\left(\frac{1}{2 n} \frac{2 \sin \frac{1}{2} h}{\sin \frac{1}{2} h+\sin \frac{1}{2}(2 n-1) h}-\frac{\sin h}{\sin h+\sin (n-1) h}\right)=O\left(\frac{1}{n^{2}}\right) .
$$

Observing that for $0<h \leq \rho \pi / n,(0<\rho<1)$

$$
\frac{\sin h}{\sin h+\sin (n-1) h}=O\left(\frac{1}{n}\right) \text {, }
$$

we obtain

$$
\begin{aligned}
I_{12} & =O\left(\frac{1}{n^{2} \sin ^{2} \frac{1}{2}\left(\theta-\theta_{k}\right)}\right)=O\left(\frac{1}{n^{2}\left(\theta-\theta_{k}\right)^{2}}\right) \\
& =O\left(\frac{1}{|k-j|^{2}}\right)=O\left(\frac{1}{i^{2}}\right) \quad(k \neq j) .
\end{aligned}
$$

Set

$$
u(x)=\frac{\sin \frac{1}{2}(2 n-x) h}{\sin \frac{1}{2} x h+\sin \frac{1}{2}(2 n-x) h}, \quad(0 \leq x \leq 2 n)
$$

Since, by [1]

$$
u^{\prime \prime}(x)=O\left(\frac{1}{n^{2}}\right)
$$

we have 


$$
I_{13}=O\left(\frac{1}{n^{3}}\right) \sum_{\jmath=2}^{2 n-2}\left|\jmath \sigma_{\jmath}\left(\theta-\theta_{k}\right)\right|=O\left(\frac{1}{n^{2} \sin ^{2} \frac{1}{2}\left(\theta-\theta_{k}\right)}\right)=O\left(\frac{1}{i^{2}}\right), \quad(k \neq \jmath) .
$$

Combining (4 13)-(4 16) yields

$$
I_{1}=O\left(\frac{1}{i^{2}}\right), \quad(k \neq j)
$$

Similarly, using that

we can prove that

$$
\sin ^{2} \frac{1}{2}\left(\theta+\theta_{k}\right) \geq \sin ^{2} \frac{1}{2}\left(\theta-\theta_{k}\right)
$$

$$
I_{2}=O\left(\frac{1}{i^{2}}\right), \quad(k \neq j) .
$$

Hence (4 9) follows from (4 11) and (4.17)-(4 18)

As to (4 10), it is a consequence of (4 7) Q.E.D

PROOF OF THEOREM 4.1. Denote by $p_{2 n-1}^{*}(x)$ the best approximation polynomial of order $\leq 2 n-1$ for function $f(x)$

$$
\left\|f(x)-p_{2 n-1}^{*}(x)\right\|_{[-1,1]}=E_{2 n-1}(f) .
$$

Then according to Theorem 2.1, we have

$$
p_{2 n-1}^{*}(x)=S_{n}\left(p_{2 n-1}^{*}, x\right) .
$$

Hence (4 5) follows from (4 7), (4.8) and the following relation

$$
f(x)-S_{n}(f, x)=f(x)-p_{2 n-1}^{*}(x)+S_{n}\left(p_{2 n-1}^{*}-f, x\right) .
$$

PROOF OF THEOREM 4.2. Since $s_{n}(1, x) \equiv \sum_{k=1}^{n} p_{k}(x)$, we have

$$
f(x)-s_{n}(f, x)=\sum_{k=1, k \neq \jmath}^{n}\left[f(x)-f\left(x_{k}\right)\right] p_{k}(x)+\left[f(x)-f\left(x_{\jmath}\right)\right] p_{\jmath}(x) .
$$

Using (4 9), (4.10), and the known results (see [4])

$$
\begin{gathered}
\left|x-x_{k}\right|=O\left(\frac{i \sqrt{1-x^{2}}}{n}+\frac{i^{2}}{n^{2}}\right), \quad(k \neq j) \\
\left|x-x_{\jmath}\right|=O\left(\frac{1}{n}\right)
\end{gathered}
$$

we can easily obtain (4.6) Q.E.D

ACKNOWLEDGEMENT. This work is supported by the Natural Sciences Foundation of Zhejiang Province

\section{REFERENCES}

[1] SUN, X.H., A new kind of interpolation: $\left(0, \delta^{M}\right)$ interpolation, submitted

[2] DeVORE, R.A., The approximation of continuous functions by positive linear operators, Springer Lecture Notes in Math., 293 (1972)

[3] POTTINGER, P., On the approximation of functions and their derivatives by Hermite interpolation, J. Approx. Theory, 23 (1978), 267-273

[4] SUN, X.H., The exact estimation of the Hermite-Fejér interpolation, J. Comput. Math., 4 (1986), 182-190, MR 87i.65019 


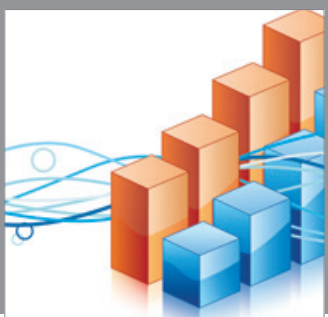

Advances in

Operations Research

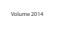

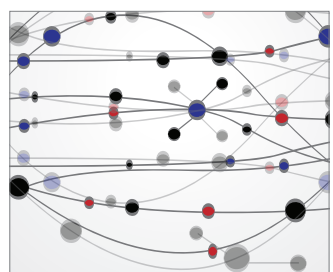

\section{The Scientific} World Journal
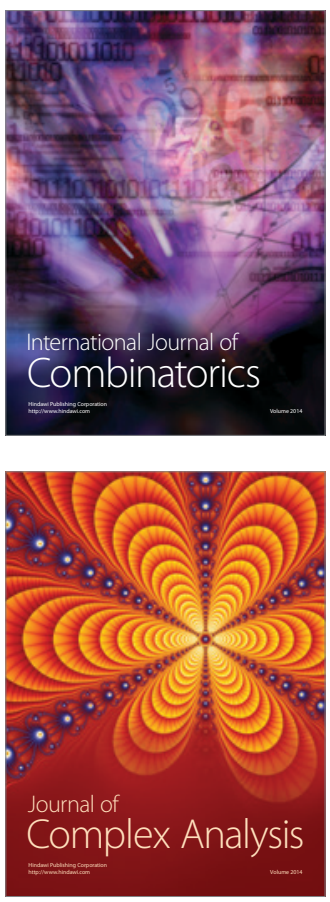

International Journal of

Mathematics and

Mathematical

Sciences
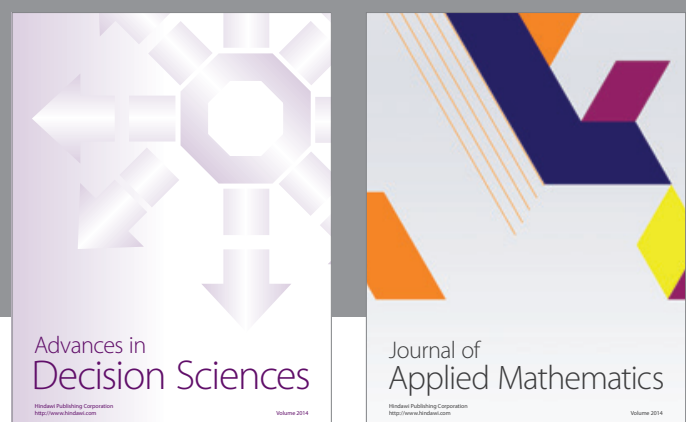

Journal of

Applied Mathematics
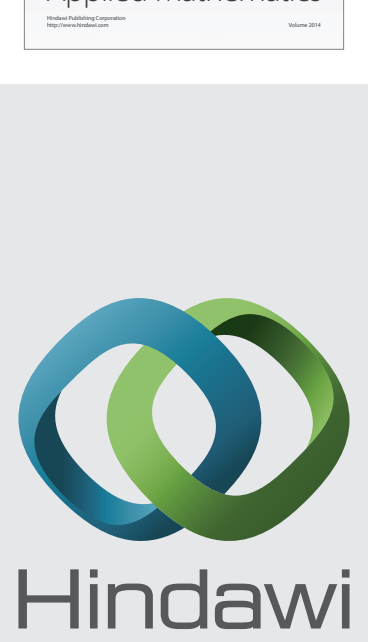

Submit your manuscripts at http://www.hindawi.com
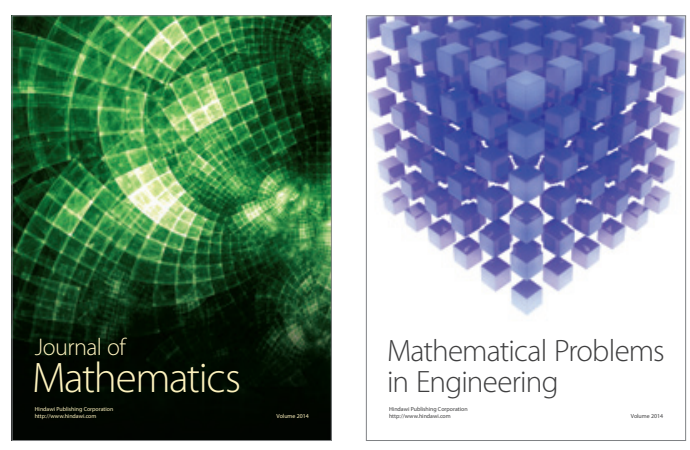

Mathematical Problems in Engineering
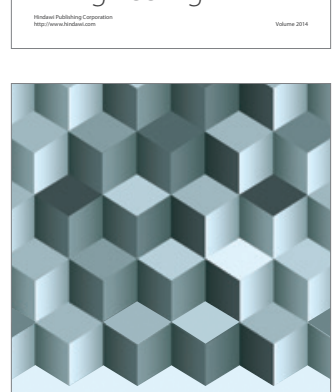

Journal of

Function Spaces
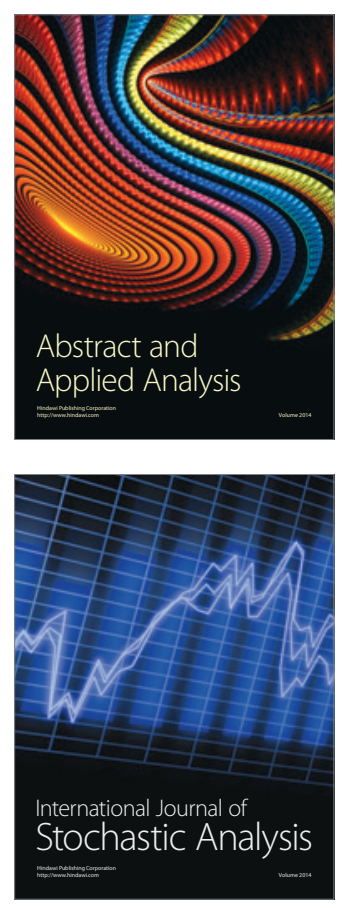

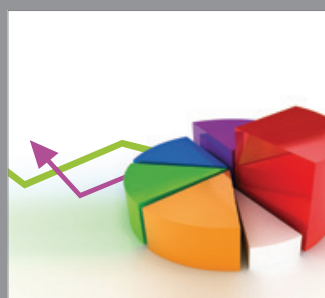

ournal of

Probability and Statistics

Promensencen
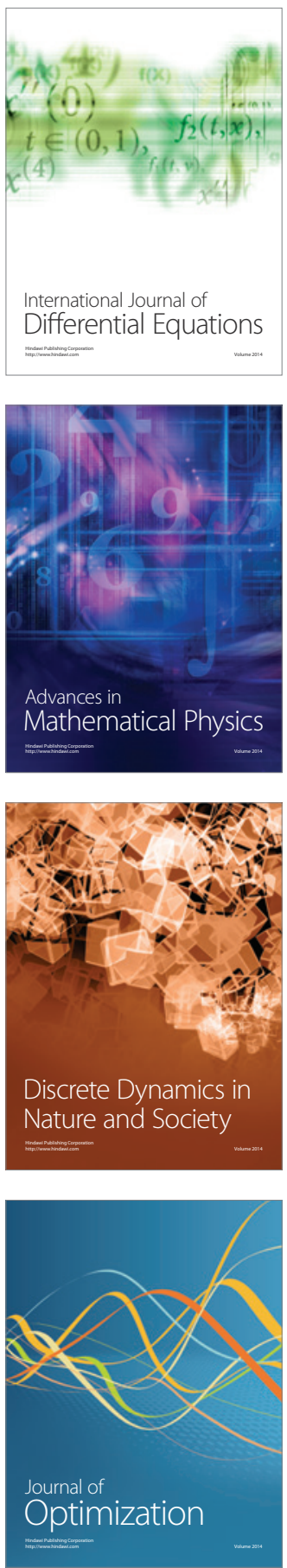